\title{
Comparison of plasma, venous and capillary blood levels of piperaquine in patients with uncomplicated falciparum malaria
}

\author{
Elizabeth A. Ashley • Kasia Stepniewska • Niklas Lindegardh • Anna Annerberg • \\ Joel Tarning • Rose McGready • Lucy Phaiphun • Pratap Singhasivanon • \\ Nicholas J. White • François Nosten
}

Received: 17 November 2009 / Accepted: 17 February 2010/Published online: 19 March 2010

(C) The Author(s) 2010. This article is published with open access at Springerlink.com

\begin{abstract}
Purpose Dihydroartemisinin-piperaquine (DP) is a fixeddose artemisinin-based combination treatment. Field pharmacokinetic studies would be simplified and facilitated by being able to use small volume capillary assays rather than venous blood. The aim of this study was to describe the relationship between piperaquine concentrations measured in capillary blood, venous blood and venous plasma.

Methods Samples of plasma, whole blood obtained by venesection and capillary blood were taken simultaneously from patients with uncomplicated Plasmodium falciparum malaria treated with DP between 0 and 9 weeks after treatment. Piperaquine concentrations in venous and capillary samples were measured using solid phase extraction and analysis by liquid chromatography with ultraviolet detection.

Results A total of 161 sets of the three measures were obtained from 54 patients. Piperaquine concentrations in
\end{abstract}

E. A. Ashley • R. McGready • L. Phaiphun • F. Nosten ( $₫)$

Shoklo Malaria Research Unit,

Mae Sot, Thailand

e-mail:smru@tropmedres.ac

K. Stepniewska $\cdot$ N. Lindegardh $\cdot$ A. Annerberg $\cdot$ J. Tarning $\cdot$

R. McGready $\cdot$ P. Singhasivanon $\cdot$ N. J. White $\cdot$ F. Nosten

Mahidol-Oxford Tropical Medicine Research Unit (MORU),

Mahidol University,

Bangkok 10400, Thailand

K. Stepniewska $\cdot$ N. Lindegardh $\cdot$ J. Tarning $\cdot$ R. McGready

N. J. White $\cdot$ F. Nosten

Centre for Tropical Medicine,

Nuffield Department of Clinical Medicine, University of Oxford,

Oxford OX3 7LJ, UK the venous blood samples were approximately twofold higher and those in the capillary blood samples were threefold higher than the corresponding venous plasma concentrations. Capillary blood piperaquine concentrations were approximately 1.7 -fold higher than venous blood concentrations, and this difference also increased with time.

Conclusion Differences in whole blood and plasma levels of piperaquine suggest compartmentalisation of the drug within blood cells, as also occurs with the structurally related quinoline chloroquine. The relationship between piperaquine concentrations in the venous plasma, venous blood and capillary blood is variable and unpredictable at low concentrations. However, within the range of concentrations usually present in patients between 3 and 21 days after treatment with currently recommended doses, the relationship between capillary and venous whole blood is predictable; consequently, capillary blood sampling can be used in field assessments.

Keywords Artemisinin combination therapy · Capillary blood $\cdot$ Malaria $\cdot$ Piperaquine

\section{Background}

The measurement of drug levels is a vital part of any comprehensive assessment of antimalarial drug efficacy. Defining the pharmacokinetic properties of the antimalarial drugs has provided a basis for optimising dosing regimens and has explained why intrinsically efficacious antimalarial drugs do not always work. Dihydroartemisinin-piperaquine (DP) is one of the leading fixed-dose artemisinin-based combination treatments (ACTs), and has recently been 
recommended as a first-line treatment for uncomplicated falciparum malaria by the World Health Organisation. Dihydroartemisinin (DHA) is a rapidly eliminated artemisinin derivative and the active metabolite of artesunate, artemether and artemotil. It has an elimination half-life of approximately $30-80 \mathrm{~min}[11,31,32]$. Piperaquine is a bisquinoline compound, structurally related to chloroquine but efficacious against chloroquine-resistant parasites. Although the drug is more than 40 years old and was used extensively in China between 1978 and 1992, estimates of the pharmacokinetic parameters of piperaquine have been published only in the last 5 years. Like chloroquine, piperaquine is extensively distributed in tissues and slowly eliminated. Terminal elimination half-life $\left(t_{1 / 2}\right)$ estimates produced by different laboratories have varied between 11 and $>60$ days $[13,14,22,28-30]$ with studies longer sampling duration and employing more sensitive assays giving longer estimates. The oral bioavailability of piperaquine has been shown to be increased by co-administration with fat, which could account for some of the observed inter-individual variation in pharmacokinetic profiles [23], although a more recent evaluation of piperaquine pharmacokinetics in Vietnamese volunteers found moderately wide variability in total drug exposure (AUC, area under the plasma concentration-time curve) which was not related to concomitant food intake [10]. Studies to date have used plasma drug assays, although results of whole blood assays at a single time point have also been reported. In two separate clinical trials in Laos [18] and Myanmar [25], piperaquine levels were measured from single samples taken on day 7 after treatment, as measurements at this time point provide a useful surrogate of in-vivo drug exposure [33]. Venous plasma levels were measured in the study in Laos and whole blood levels (taken onto filter paper) were analysed in the Myanmar study. The mean [95\% confidence limit $(95 \% \mathrm{CI})$ ] day 7 plasma piperaquine concentration in the Laos study was $31.8(28.8-34.8) \mathrm{ng} / \mathrm{ml} \quad(n=104)$, whereas in the Myanmar study, the mean capillary whole blood value was $72 \mathrm{ng} / \mathrm{ml}$ [median (interquartile range) 62 $(50-82) \mathrm{ng} / \mathrm{ml}](n=156)$. Thus, concentrations assessed from capillary whole blood were approximately double those measured in plasma at the same time point.

The requirements for multiple venepunctures and a cold chain to transport samples to an analytical laboratory are major impediments in conducting community-based studies of antimalarial drugs that incorporate the measurement of drug concentrations. Assays have been adapted to the field with the development of sensitive methods capable of measuring concentrations in very small volume capillary blood samples, often taken onto filter paper, and by the use of sparse sampling schedules coupled with non-linear mixed-effects modelling to generate pharmacokinetic estimates and investigate sources of variation [24].
The principal aim of this study was to compare piperaquine concentrations in samples of venous plasma and venous whole blood with those in capillary whole blood taken simultaneously and to determine the relationship between them.

\section{Study design and methods}

Study site and population

This was part of a detailed pharmacokinetic study nested into two larger randomised trials of the efficacy and safety of DP which have been reported previously $[1,2,30]$. It took place in clinics of the Shoklo Malaria Research Unit along the Thai-Myanmar border.

Karen or Burmese patients, aged $>2$ years, with symptomatic uncomplicated falciparum infection, randomised to treatment with DP and to the nested pharmacokinetic study, were eligible for inclusion. The study was explained to patients in their own language, and written consent was obtained (thumbprint if patients were unable to read or write).

\section{DP dosing regimen}

Treatment was directly observed. The total dose was $7 \mathrm{mg} /$ $\mathrm{kg}$ body weight of DHA and $55 \mathrm{mg} / \mathrm{kg}$ piperaquine (Artekin Holley Pharmaceutical Co, Guangzhou, China) split into four doses at $0,8,24 \mathrm{~h}$ and $48 \mathrm{~h}$, respectively, or three doses at 0,24 and $48 \mathrm{~h}$, respectively.

\section{Sampling}

A maximum of four sets of post-treatment samples was collected from each patient. These were selected randomly from the following time windows after the first dose of DP: $0-4,8-12,24-48,48-52 \mathrm{~h}$ (three-dose arm) or 4-8, 12-24, 28-48, 52-72 h (four-dose arm), and 7, 14, 21, 28, 35, 42, 49, 56 or 63 days (both groups). From the $4.5-\mathrm{ml}$ blood sample taken by venesection, $1.5 \mathrm{ml}$ was transferred to a cryo tube, and the remainder was transferred into a sodium heparin tube; this sample was centrifuged for $10 \mathrm{~min}$ at $1500 \mathrm{~g}$ and the plasma transferred by pipette to a cryo tube before freezing in liquid nitrogen. Capillary samples were taken at the same time from a fingerprick into heparinised capillary tubes. The blood was then transferred to a cryo tube to be frozen immediately in liquid nitrogen. Samples were transferred to the main laboratory in batches where they were stored at $-80^{\circ} \mathrm{C}$. After all samples were collected, they were transferred on dry ice to the Pharmacology Laboratory in the Faculty of Tropical Medicine, Mahidol University, Bangkok. Piperaquine concentrations were measured using a high-throughput assay utilising solid 
phase extraction (SPE) and liquid chromatography with ultra-violet detection [15-17]. The lower limit of quantification (LLOQ) was higher for the blood assays than for the plasma assay, mainly because of differences in sample volumes but also because the recovery of piperaquine from blood is slightly lower than that from plasma. The plasma assay could accommodate $1 \mathrm{ml}$ while the blood assay only accommodated $500 \mu \mathrm{L}$. A larger volume of blood resulted in an unacceptably high back-pressure in the SPE step. The plasma assay used a 1-ml sample, the blood assay used $0.5 \mathrm{ml}$ and the capillary blood assay used $0.1 \mathrm{ml}$. The sample volumes and the impact on assay sensitivity are summarised in Table 1.

\section{Statistical analysis}

The relationships between piperaquine concentrations in the venous plasma and venous blood, venous plasma and capillary blood and capillary blood and venous blood were investigated using regression modelling. Since all concentrations were log-normally distributed, they were modelled after a logarithmic transformation. The relationship between the concentrations was modelled as a power function, and the optimal fractional polynomial function was found using the 'fracpoly' command in Stata ver. 10 (StataCorp, College Station, TX). The final model was a random intercept model to account for multiple measurements per subject and was adjusted for time since the first dose or log-parasitaemia, if either improved the model significantly, as assessed by the Wald test. For example, the relationship between venous plasma and venous whole blood concentrations was estimated using the following model structure:

$$
\begin{aligned}
\log (C \text { venous blood })_{i j}=\beta & +F P\left(\log (C \text { venous plasma })_{i j}\right) \\
& +\beta_{1} x_{1 i j}+\beta_{2} x_{2 i j}+v_{i}+e_{i j}
\end{aligned}
$$

where $\mathrm{FP}(\mathrm{x})$ is a fractional polynomial $\alpha_{1} \mathrm{x}^{\mathrm{p} 1}+\ldots+\alpha_{\mathrm{m}} \mathrm{x}^{\mathrm{pm}}$; $\mathrm{p}_{1}<\ldots<\mathrm{p}_{\mathrm{m}}$ are integer or fractional powers; $\mathrm{x}_{1}$ and $\mathrm{x}_{2}$ are other covariates, such as time from the first dose or log of parasitaemia on the day of sampling, $\mathrm{v}_{\mathrm{i}} \sim \mathrm{N}\left(0, \delta_{\mathrm{b}}\right)$ is a subjectspecific residual and $\mathrm{e}_{\mathrm{ij}} \sim \mathrm{N}(0, \delta)$ is a usual residual. Parasitaemia at the time of sampling was estimated from the fitted log-linear decline in parasite densities assuming a firstorder elimination process. For fitting purposes parasitaemia was assumed to be equal to half of the detection limit for the first negative slide.

Normal plots of residuals were examined to check the assumptions of the regression model. Bland-Altman plots were used to assess differences between predicted and measured concentrations. The utility of the models was assessed by examining the relative difference between the predicted and observed (measured) concentrations on the original scale; for example: (predicted venous plasma concentration - measured venous plasma concentration)/ measured venous plasma concentration $\times 100 \%$.

\section{Ethical approval}

Approval for the study was granted by the Faculty of Tropical Medicine Ethical Committee, Mahidol University, Bangkok, Thailand and the Oxford Tropical Ethics Committee (OXTREC), UK.

\section{Results}

The study took place between 2003 and 2004. A total of 161 sets of three simultaneous measures from 54 patients were analysed. The patient characteristics are shown in Table 2. A median of three (range 1-4) sets of samples were collected from the same patient. Piperaquine was detectable in all of the plasma samples but undetectable in three venous blood samples and 20 capillary blood samples. The median piperaquine concentrations were 136.1 (range $0-907.4) \mathrm{ng} / \mathrm{ml}$ in capillary samples, $83.9(0-852.5) \mathrm{ng} / \mathrm{ml}$ in venous samples and $36.3(2.8-345.1) \mathrm{ng} / \mathrm{ml}$ in venous plasma samples. The LLOQ of the assays, coefficients of

\begin{tabular}{|c|c|c|c|c|c|c|c|c|c|}
\hline \multirow{2}{*}{$\frac{\text { Parameters }}{\text { Control concentration }(\mathrm{ng} / \mathrm{ml})}$} & \multicolumn{3}{|c|}{ Venous plasma ( $n=486$ samples) } & \multicolumn{3}{|c|}{ Venous blood ( $n=186$ samples) } & \multicolumn{3}{|c|}{ Capillary blood ( $n=178$ samples) } \\
\hline & 20.0 & 100 & 613 & 59.6 & 400 & 2000 & 59.6 & 400 & 2000 \\
\hline Mean value & 19.6 & 98.0 & 636 & 56.4 & 409.3 & 1979 & 59.3 & 410.5 & 2113 \\
\hline $\mathrm{SD}$ & 1.3 & 4.1 & 26 & 6.5 & 37.0 & 110 & 4.9 & 14.1 & 127 \\
\hline RSD (\%) & 6.7 & 4.1 & 4.1 & 11.6 & 9.0 & 5.5 & 8.3 & 3.4 & 6.0 \\
\hline Sample volume $(\mu \mathrm{l})$ & \multicolumn{3}{|l|}{1000} & \multicolumn{3}{|l|}{500} & \multicolumn{3}{|l|}{100} \\
\hline LLOQ (ng/ml) & \multicolumn{3}{|l|}{2.5} & \multicolumn{3}{|l|}{15} & \multicolumn{3}{|l|}{37.33} \\
\hline
\end{tabular}

Table 1 Assay sensitivity and quality control results

SD, standard deviation; RSD, relative standard deviation; LLOQ, lower limit of quantification

There were more plasma samples than venous blood and capillary blood samples because not all patients in the larger pharmacokinetic study gave all types of blood for testing 
Table 2 Characteristics of 97 patients with uncomplicated falciparum malaria treated with dihydroartemisinin-piperaquine (DP) in the pharmacokinetic study

\begin{tabular}{ll}
\hline Patient characteristics & Values \\
\hline Male, $n(\%)$ & $58(60)$ \\
Age (years) & $25(3-55)$ \\
Weight $(\mathrm{kg})$ & $48(12-74)$ \\
Haematocrit on enrolment (\%) & $39(20-52)$ \\
Three-dose DP treatment, $n(\%)$ & $47(49)$ \\
Parasitaemia/ $\mu \mathrm{l}$, geometric mean & $10,887(83-223,872)$ \\
Piperaquine total dose, $\mathrm{mg} / \mathrm{kg}$ & $53.3(40-74)$ \\
\hline
\end{tabular}

The results are expressed as the median value with the range given in parenthesis unless stated otherwise

variation and the quality control results are shown in Table 1.

Venous blood versus venous plasma

There were three pairs of samples for which piperaquine was below the limit of detection in venous blood but detectable in plasma taken at the same time (plasma concentrations $4.3,4.8$ and $40.0 \mathrm{ng} / \mathrm{ml}$, respectively). For the other paired concentrations, venous blood concentrations were nearly always higher than venous plasma concentrations with a median $(90 \%$ range) ratio of venous blood concentration to venous plasma concentration of 2.15
(0.91-5.26). This ratio was strongly negatively correlated with plasma concentration (Spearman's $\rho=-0.578, p<$ 0.001 ) and parasitaemia on the day of sampling (Spearman's $\rho=-0.417, p<0.001$ and positively correlated with the interval between dosing and sampling (Spearman's $\rho=$ $0.550, p<0.001)$. The best model to describe the relationship between venous blood log-concentration and venous plasma log-concentration was linear and included logparasitaemia at the time of sampling $(p<0.001)$ (Table 3). The fit was considerably worse at low plasma concentrations, particularly if the time and parasite covariates were not included (Fig. 1a). A Bland-Altman plot (Fig. 1b) shows a small decrease in the difference between observed and predicted log concentrations [by 0.109 (95\% CI 0.033 0.184 per $1 \mathrm{U})$ ], which corresponds to much larger differences on the original scale.

Venous plasma versus capillary blood

In 20 pairs of samples piperaquine was undetectable in capillary blood but detectable in plasma taken at the same time [median plasma concentrations 4.8 (90\% range 2.940.0) $\mathrm{ng} / \mathrm{ml}$. Median capillary blood levels at which piperaquine was detectable in both venous plasma and capillary blood samples were 3.5 -fold (90\% range 1.7-7.4) higher than those measured in plasma. This ratio was not correlated with plasma concentration, the time of sampling or parasitaemia on the day of sampling. The best model to describe the relationship between log plasma concentration
Table 3 Summary of the models used to describe the relationship between the concentrations of piperaquine in venous plasma and those in capillary and venous whole blood $\sigma_{\mathrm{b}}$, between subject variation; $\sigma$, residual variation

${ }^{\mathrm{a}}$ Likelihood ratio test of $\sigma_{\mathrm{b}}=0$

\begin{tabular}{|c|c|c|c|c|}
\hline Parameters & Transformation & Coefficient & $95 \%$ Confidence interval & $p$ value \\
\hline \multicolumn{5}{|c|}{$\log ($ venous blood concentration $)=\mathrm{f}($ venous plasma concentration $)$} \\
\hline Plasma concentration & $\log (\mathrm{x})$ & 0.754 & $0.697-0.811$ & 0.000 \\
\hline Parasitaemia & $\log _{10}(x+1)$ & -0.125 & -0.167 to -0.082 & 0.001 \\
\hline Constant $(\alpha)$ & & 1.752 & $1.538-1.966$ & 0.000 \\
\hline$\sigma_{\mathrm{b}}$ & & 0.210 & $0.138-0.319$ & $0.005^{\mathrm{a}}$ \\
\hline$\sigma$ & & 0.358 & $0.312-0.410$ & \\
\hline$\rho=\sigma_{b}^{2} /\left(\sigma_{b}^{2}+\sigma^{2}\right)$ & & 0.256 & $0.109-0.468$ & \\
\hline \multicolumn{5}{|c|}{$\log ($ venous plasma concentration $)=\mathrm{f}($ capillary blood concentration $)$} \\
\hline Capillary concentration & $\log (x)$ & 0.974 & $0.864-1.084$ & $<0.001$ \\
\hline Constant $(\alpha)$ & & -1.072 & -1.637 to -0.506 & $<0.001$ \\
\hline$\sigma_{\mathrm{b}}$ & & 0.294 & $0.192-0.449$ & $0.002^{\mathrm{a}}$ \\
\hline$\sigma$ & & 0.456 & $0.391-0.530$ & \\
\hline$\rho=\sigma_{b}^{2} /\left(\sigma_{b}^{2}+\sigma^{2}\right)$ & & 0.293 & $0.125-0.525$ & \\
\hline \multicolumn{5}{|c|}{$\log ($ venous blood concentration $)=\mathrm{f}($ capillary blood concentration $)$} \\
\hline Capillary concentration & $\log (\mathrm{x})$ & 0.920 & $0.903-0.938$ & $<0.001$ \\
\hline Parasitaemia & $\log _{10}(\mathrm{x}+1)$ & -0.112 & -0.145 to -0.078 & $<0.001$ \\
\hline Constant $(\alpha)$ & 0 & & & \\
\hline$\sigma_{\mathrm{b}}$ & & 0.252 & $0.189-0.338$ & $<0.001^{\mathrm{a}}$ \\
\hline$\sigma$ & & 0.264 & $0.228-0.306$ & \\
\hline$\rho=\sigma_{b}^{2} /\left(\sigma_{b}^{2}+\sigma^{2}\right)$ & & 0.477 & $0.306-0.653$ & \\
\hline
\end{tabular}



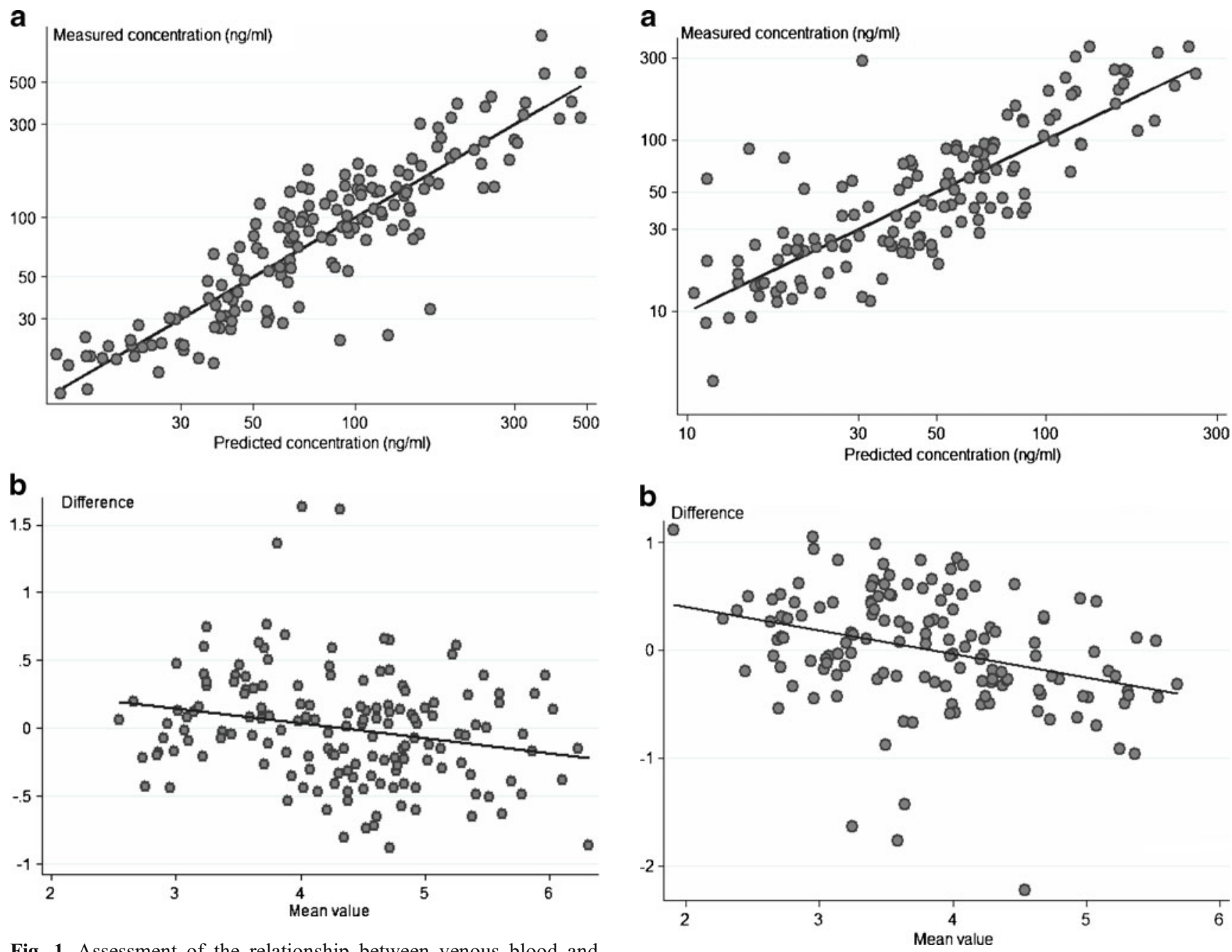

Fig. 1 Assessment of the relationship between venous blood and venous plasma concentrations of piperaquine. Model was adjusted for parasitaemia at the time of sampling. a Predicted versus measured venous blood concentrations. Natural log scale. b BlandAltman plot for measured and predicted venous blood concentration

and $\log$ capillary concentration was linear without covariates (Table 3). Predicted (from piperaquine capillary concentrations) versus measured piperaquine venous plasma concentrations are shown in Fig. 2a. The Bland-Altman plot (Fig. 2b) shows a decrease in the difference between observed and predicted log concentrations [by $0.218(90 \%$ range $0.113-0.323)$ per $1 \mathrm{U}$ ], which corresponds to much larger differences on the original scale.

\section{Capillary blood versus venous blood}

Piperaquine was undetectable in 20 capillary blood samples; of these, piperaquine was also undetectable in three corresponding samples of venous blood. Of the remaining 17 samples, the median venous concentration ( $90 \%$ range) was $20.55(12.32-68.65) \mathrm{ng} / \mathrm{ml}$.

On average, capillary drug levels were 1.66-fold higher (90\% range $0.92-3.03$ ) than the levels measured in

Fig. 2 Assessment of the relationship between venous plasma and capillary blood concentrations of piperaquine. a Predicted versus measured venous plasma concentrations. Natural log scale. b BlandAltman plot for measured and predicted venous plasma concentration

corresponding venous blood samples. This ratio was strongly negatively correlated with the interval between dosing and sampling (Spearman's $\rho=-0.45, p<0.001$ ) and positively correlated with parasitaemia on the day of sampling (Spearman's $\rho=0.45, p<0.001)$. The best model to describe the relationship between venous log-concentration and capillary log-concentration was linear and included log-parasitaemia at the time of sampling $(p<0.001)$ (Table 3). The BlandAltman plot (Fig. 3) shows a small decrease in the difference between observed and predicted log concentrations [by $0.128(90 \%$ range $0.052-0.204)$ per $1 \mathrm{U}$ ] which would be 0.175 (90\% range $0.92-0.258)$ if parasitaemia was not included in the model. Differences on the original scale are larger, but the majority $(89 \%, 125 / 141)$ of measures remain within $50 \%$. Overall, $58 \%(82 / 141)$ of predicted venous blood concentrations (from capillary concentrations) were within $20 \%$ of the measured venous concentrations, while $70 \%(99 / 141)$ were within $30 \%$. The discrepancies were 

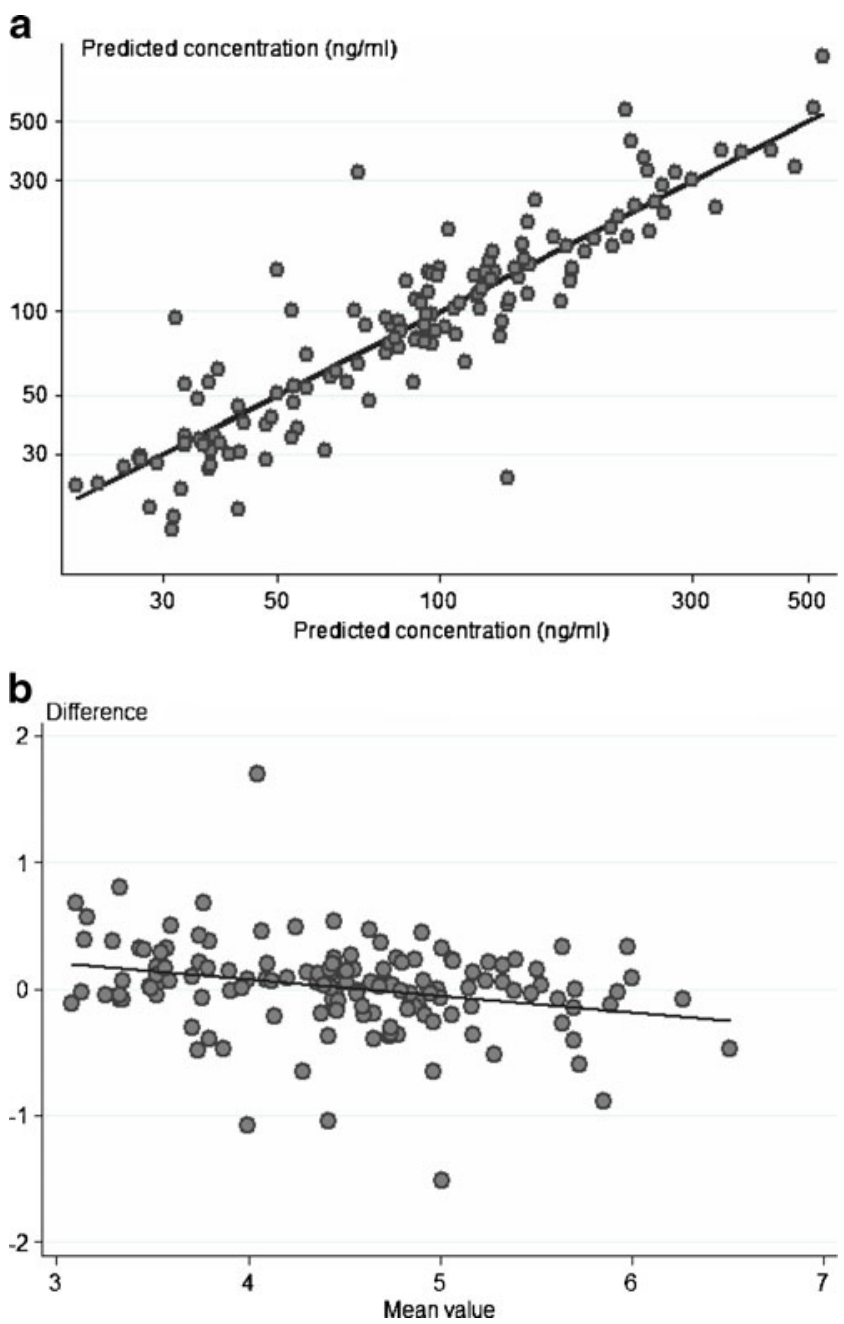

Fig. 3 Assessment of the relationship between venous blood and capillary blood concentrations of piperaquine. Model was adjusted for parasitaemia at the time of sampling. a Predicted versus measured venous blood concentrations. Natural log scale. b BlandAltman plot for measured and predicted venous blood concentration

higher for low concentrations, with only $41 \%$ (7/17) of predicted venous blood concentrations remaining within the 20 or $30 \%$ range for venous concentrations $<30 \mathrm{ng} / \mathrm{ml}$. For concentrations $>30 \mathrm{ng} / \mathrm{ml}$, these proportions were $60 \%(75 /$ $124)$ and $74 \%(92 / 124)$, respectively. These values remained the same if the cut-off was taken as 40 or $50 \mathrm{ng} / \mathrm{ml}$. Nearly all $(93 \%$, or $96 / 103)$ capillary blood concentrations taken between $24 \mathrm{~h}$ after the first dose and before or on day 21 were $>30 \mathrm{ng} / \mathrm{ml}$. From day 28 onwards, the majority $(77 \%)$ of concentrations were $<30 \mathrm{ng} / \mathrm{ml}$. After day 3, when parasitaemia had cleared, the relationship between venous piperaquine concentrations and capillary concentrations became simpler: venous concentration $=$ (capillary concentration $)^{0.9}$. Among samples taken after day 3 and before day 28 , $70 \%(42 / 60)$ of the predicted venous blood concentrations were within $30 \%$ of the measured venous blood concentrations, and $62 \%(37 / 60)$ of predictions were within $20 \%$; consequently, the observed versus expected agreement was good in this time interval.

Figure 4 compares the predictive utilities of all models.

\section{Discussion}

In most pharmacological assessments drugs are measured in plasma. Many antimalarial drugs are eliminated slowly from the body, and the measurement of drug concentrations in small volumes of whole blood taken from a finger prick sample is more convenient and more acceptable in young children. This allows community-based assessments, even in very remote settings, providing essential information on drug efficacy, adherence and toxicity. The new ACT dihydroartemisinin-piperaquine is likely to have a major role in antimalarial treatment, so the development of fieldbased tools for its assessment is important. The results of this study show that measurements of piperaquine in venous plasma, venous or capillary blood are not readily interchangeable across the whole range of concentrations encountered in patients with current dosing. Piperaquine concentrations were highest in capillary whole blood, followed in decreasing concentrations by venous whole blood and venous plasma. The higher concentrations of piperaquine detected in whole blood compared to plasma suggests that piperaquine concentrates within blood cells. These results are consistent with previous findings that capillary blood concentrations were approximately double

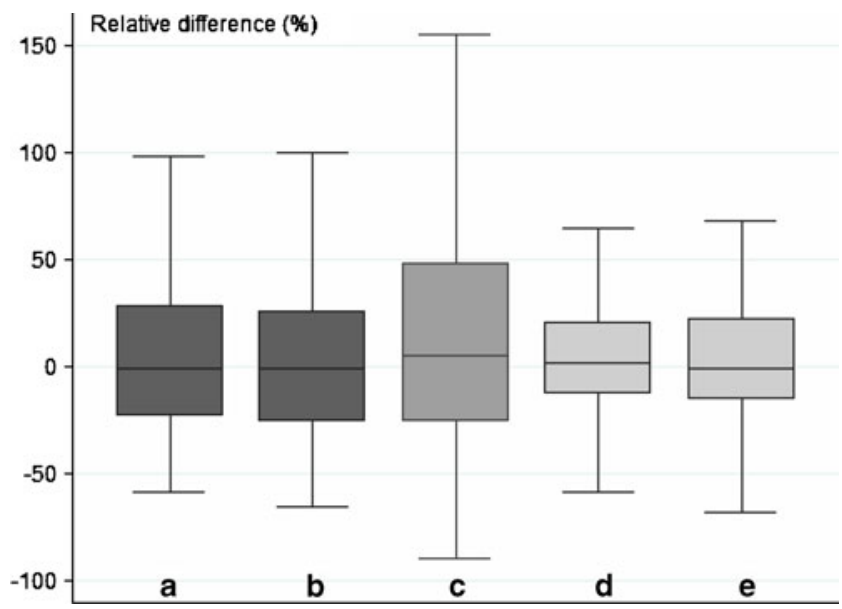

Fig. 4 Distribution of the relative difference (\%) between measured and predicted values of piperaquine for: a venous blood concentration predicted from venous plasma concentration, adjusted for parasitaemia, b venous blood concentration predicted from venous plasma concentration, unadjusted for parasitaemia, $\mathbf{c}$ venous plasma concentration predicted from capillary blood concentration, $\mathbf{d}$ venous blood concentration predicted from capillary blood concentration, adjusted for parasitaemia, e venous blood concentration predicted from capillary blood concentration, unadjusted for parasitaemia. Boundaries of the boxes 25 th and 75 th centiles, line in the box median 
those measured in plasma at day $7[18,25]$. Studies of chloroquine pharmacokinetics have reported concentrations in erythrocytes that are usually two to fivefold higher than those in plasma. Chloroquine is also concentrated markedly in leukocytes and platelets [3]. This underlies the differences between plasma and serum concentrations and contributes to the variability in plasma concentrations. The duration and force of centrifugation has been shown to influence the concentrations of chloroquine and its metabolite desethylchloroquine in plasma, with concentrations of both drugs decreasing as the centrifugal force increases until relatively stable levels are reached at $500 \mathrm{~g}$ (due to variable removal of white cells and platelets). The measurement of whole blood concentrations avoids this potential confounder [21] and is now the generally accepted method for measuring chloroquine in therapeutic assessments. In the seminal study of Gustafasson et al. [9] of 11 healthy volunteers in whom chloroquine concentrations were measured in plasma and erythrocytes for 3-8 weeks after dosage, the disposition of chloroquine in erythrocytes paralleled that in plasma.

There is a consistent increase in platelet count (often doubling) and haematocrit (circa 10\%) in patients recovering from acute uncomplicated malaria [20]. Neutrophil counts also tend to increase. In acute malaria, patients may be dehydrated and haemoconcentrated when they present, and there are reduced concentrations of albumin (which binds acidic drugs) and marked increases in the concentrations of the acute phase protein $\alpha 1$-acid glycoprotein (binds basic drugs). In addition, capillary blood samples are affected by factors affecting peripheral perfusion and tissue fluid concentrations as they contain a variable admixture of interstitial fluid. In cases of acute malaria, patients are usually febrile and vasodilated, whereas following recovery there may be peripheral vasoconstriction, leading to greater tissue fluid admixture in capillary samples. Previous studies in patients and volunteers without malaria have revealed that the haematocrit and leucocyte levels tend to be higher in capillary blood samples than in venous blood samples, while platelet numbers tend to be similar or lower [5-7,27, 34]. Haematocrit measurements have also been observed to be more variable on repeated capillary sampling than on repeated venous sampling. This could partially explain the inter-individual variability of the relationship shown here. For certain drugs, such as neuroleptics, the red cell concentrations have been reported to correlate better with therapeutic effects or dose than plasma concentrations $[4,8$, 26].

Hinderling [12] has argued that for drugs with a $\mathrm{Kb} / \mathrm{p}$ (blood to plasma concentration ratio) $>2.0$, measuring concentrations in whole blood or erythrocytes rather than in plasma lowers the LLOQ. The increased sensitivity permits the kinetics of the drug to be followed up for at least one additional half-life. In our study, the LLOQ was higher in the blood assays than the plasma assay, mainly because of differences in sample volumes but also because the recovery of piperaquine from blood is slightly lower than that from plasma. The plasma assay used a $1-\mathrm{ml}$ sample to obtain a LLOQ of $2.5 \mathrm{ng} / \mathrm{ml}$, while the capillary blood assay used a $0.1-\mathrm{ml}$ sample to obtain a LLOQ of $37 \mathrm{ng} / \mathrm{ml}$.

The model for the relationship between piperaquine concentrations measured in venous and capillary blood adjusted for parasitaemia showed the least amount of variability between observed and predicted concentrations, but this may be attributable to the large proportion of undetectable results at the lower end of the concentration range for the capillary measurements.

The relationship between piperaquine concentrations in plasma, venous blood and capillary bloods is variable and not predictable at low concentrations. Further studies are recommended to characterise the compartmentalisation in blood cells, to compare estimates of piperaquine pharmacokinetic parameters in plasma and whole blood and to provide information on the optimum samples for field studies in terms of drug measurement. A simple approach for most field studies is to take a single measurement of drug concentration on day 7. At this point, parasitaemia has almost invariably cleared, and a good approximation of the venous concentration is (capillary concentration) ${ }^{0.9}$. Thus, despite certain limitations, the capillary blood piperaquine measurement could prove to be useful in field assessment studies of therapeutic efficacy and adherence.

Acknowledgments We would like to thank all the patients of SMRU who provided samples for the study and all colleagues involved in taking, processing, transporting and analysing specimens. This work was part of the Wellcome Trust-Mahidol University, Oxford Tropical Medicine Research Programme, funded by the Wellcome Trust.

Open Access This article is distributed under the terms of the Creative Commons Attribution Noncommercial License which permits any noncommercial use, distribution, and reproduction in any medium, provided the original author(s) and source are credited.

\section{References}

1. Ashley EA, Krudsood S, Phaiphun L, Srivilairit S, McGready R, Leowattana W, Hutagalung R, Wilairatana P, Brockman A, Looareesuwan S, Nosten F, White NJ (2004) Randomized, controlled dose-optimization studies of dihydroartemisininpiperaquine for the treatment of uncomplicated multidrugresistant falciparum malaria in Thailand. J Infect Dis 190 (10):1773-1782, Erratum in: J Infect Dis. 2005;191(7):1204

2. Ashley EA, McGready R, Hutagalung R, Phaiphun L, Slight T, Proux S, Thwai KL, Barends M, Looareesuwan S, White NJ, Nosten F (2005) A randomized, controlled study of a simple, once-daily regimen of dihydroartemisinin-piperaquine for the 
treatment of uncomplicated, multidrug-resistant falciparum malaria. Clin Infect Dis 41(4):425-432

3. Bergqvist Y, Domeij-Nyberg B (1983) Distribution of chloroquine and its metabolite desethyl-chloroquine in human blood cells and its implication for the quantitative determination of these compounds in serum and plasma. J Chromatogr 272 (1): $137-148$

4. Casper R, Garver DL, Dekirmenjian H, Chang S, Davis J (1980) Phenothiazine levels in plasma and red blood cells. Arch Gen Psychiatry 37:301-305

5. Daae LN, Halvorsen S, Mathisen PM, Mironska K (1988) A comparison between haematological parameters in 'capillary' and venous blood from healthy adults. Scand J Clin Lab Invest 48(7):723-726, Erratum in: Scand J Clin Lab Invest 1989;49 (3):305

6. Daae LN, Hallerud M, Halvorsen S (1991) A comparison between haematological parameters in 'capillary' and venous blood samples from hospitalized children aged 3 months to 14 years. Scand J Clin Lab Invest 51(7):651-654

7. Feusner JH, Behrens JA, Detter JC, Cullen TC (1979) Platelet counts in capillary blood. Am J Clin Pathol 72(3):410-414

8. Garver DL, Dekirmenjian H, Davis JM, Casper R, Ericksen S (1977) Neuroleptic drug levels and therapeutic response: preliminary observations with red blood cells - bound butaperazine. Am J Psychiatry 134:304-307

9. Gustafasson LL, Walker O, Alván G, Beermann B, Estevez F, Gleisner L, Lindström B, Sjöqvist F (1983) Disposition of chloroquine in man after single intravenous and oral doses. $\mathrm{Br} \mathrm{J}$ Clin Pharmacol 15(4):471-479

10. Hai TN, Hietala SF, Van Huong N, Ashton M (2008) The influence of food on the pharmacokinetics of piperaquine in healthy Vietnamese volunteers. Acta Trop 107(2):145-149

11. Hien TT, Davis TM, Chuong LV, Ilett KF, Sinh DX, Phu NH, Agus C, Chiswell GM, White NJ, Farrar J (2004) Comparative pharmacokinetics of intramuscular artesunate and artemether in patients with severe falciparum malaria. Antimicrob Agents Chemother 48(11):4234-4239, Erratum in: Antimicrob Agents Chemother. 2005;49(2):871

12. Hinderling PH (1997) Red blood cells: a neglected compartment in pharmacokinetics and pharmacodynamics. Pharmacol Rev 49 (3):279-295

13. Hung TY, Davis TM, Ilett KF (2003) Measurement of piperaquine in plasma by liquid chromatography with ultraviolet absorbance detection. J Chromatogr B Analyt Technol Biomed Life Sci 791 (1-2):93-101

14. Hung TY, Davis TM, Ilett KF, Karunajeewa H, Hewitt S, Denis MB, Lim C, Socheat D (2004) Population pharmacokinetics of piperaquine in adults and children with uncomplicated falciparum or vivax malaria. Br J Clin Pharmacol 57(3):253-262

15. Lindegardh N, Ashton M, Bergqvist Y (2003) Automated solidphase extraction method for the determination of piperaquine in whole blood by rapid liquid chromatography. Ther Drug Monit 25 (5):544-551

16. Lindegardh N, Ashton M, Bergqvist Y (2003) Automated solidphase extraction method for the determination of piperaquine in plasma by peak compression liquid chromatography. J Chromatogr Sci 41(1):44-49

17. Lindegardh N, White NJ, Day NP (2005) High throughput assay for the determination of piperaquine in plasma. J Pharm Biomed Anal 39(3-4):601-605

18. Mayxay M, Thongpraseuth V, Khanthavong M, Lindegardh N, Barends M, Keola S, Pongvongsa T, Phompida S, Phetsouvanh R, Stepniewska K, White NJ, Newton PN (2006) An open, randomized comparison of artesunate plus mefloquine vs. dihydroartemisinin- piperaquine for the treatment of uncomplicated Plasmodium falciparum malaria in the Lao People's Democratic Republic (Laos). Trop Med Int Health 11(8):1157-1165

19. Orjih AU (1987) Increased accumulation of chloroquine and desethylchloroquine in homozygous sickle cells. Bull WHO 65 (1):51-55

20. Price RN, Simpson JA, Nosten F, Luxemburger C, Hkirjaroen L, ter Kuile F, Chongsuphajaisiddhi T, White NJ (2001) Factors contributing to anemia after uncomplicated falciparum malaria. Am J Trop Med Hyg 65(5):614-622

21. Rombo L, Ericsson O, Alvan G, Lindstrom B, Gustafsson LL, Sjoqvist F (1985) Chloroquine and desethylchloroquine in plasma, serum, and whole blood: problems in assay and handling of samples. Ther Drug Monit 7(2):211-215

22. Roshammar D, Hai TN, Friberg Hietala S, Van Huong N, Ashton M (2006) Pharmacokinetics of piperaquine after repeated oral administration of the antimalarial combination CV8 in 12 healthy male subjects. Eur J Clin Pharmacol 62(5):335-341

23. Sim IK, Davis TM, Ilett KF (2005) Effects of a high-fat meal on the relative oral bioavailability of piperaquine. Antimicrob Agents Chemother 49(6):2407-2411

24. Simpson JA, Aarons L, White NJ (2001) How can we do pharmacokinetic studies in the tropics? Trans R Soc Trop Med Hyg 95:347-351

25. Smithuis F, Kyaw MK, Phe O, Aye KZ, Htet L, Barends M, Lindegardh N, Singtoroj T, Ashley E, Lwin S, Stepniewska K, White NJ (2006) Efficacy and effectiveness of dihydroartemisininpiperaquine versus artesunate-mefloquine in falciparum malaria: an open-label randomized comparison. Lancet 367(9528):2075-2085

26. Svensson C, Nyberg G, Axelsson R, Mårtensson E (1986) Concentrations of thioridazine and thioridazine metabolites in erythrocytes. Psychopharmacology 89:291-293

27. Tai DY, Chan KW, Chee YC, Mak KH (1995) Comparison of platelet counts in simultaneous venous and capillary blood samples using an automated platelet analyser. Singapore Med J 36(3):263-266

28. Tarning J, Bergqvist Y, Day NP, Bergquist J, Arvidsson B, White NJ, Ashton M, Lindegardh N (2006) Characterization of human urinary metabolites of the antimalarial piperaquine. Drug Metab Dispos 34(12):2011-2019

29. Tarning J, Lindegardh N, Annerberg A, Singtoroj T, Day NP, Ashton M, White NJ (2005) Pitfalls in estimating piperaquine elimination. Antimicrob Agents Chemother 49(12):5127-5128

30. Tarning J, Ashley EA, Lindegardh N, Stepniewska K, Phaiphun L, Day NP, McGready R, Ashton M, Nosten F, White NJ (2008) Population pharmacokinetics of piperaquine after two different treatment regimens with dihydroartemisinin-piperaquine in patients with acute uncomplicated Plasmodium falciparum malaria in Thailand. Antimicrob Agents Chemother 52(3):1052-1061, 2007

31. Teja-Isavadharm P, Watt G, Eamsila C, Jongsakul K, Li Q, Keeratithakul G, Sirisopana N, Luesutthiviboon L, Brewer TG (2001) Kyle DE.Comparative pharmacokinetics and effect kinetics of orally administered artesunate in healthy volunteers and patients with uncomplicated falciparum malaria. Am J Trop Med Hyg 65(6):717-721

32. White NJ (1994) Clinical pharmacokinetics and pharmacodynamics of artemisinin and derivatives. Trans R Soc Trop Med Hyg 88 [Suppl 1]:S41-S43

33. White NJ, Stepniewska K, Barnes K, Price RN, Simpson J (2008) Simplified antimalarial therapeutic monitoring: using the day-7 drug level? Trends Parasitol 24:159-163

34. Yang ZW, Yang SH, Chen L, Qu J, Zhu J, Tang Z (2001) Comparison of blood counts in venous, fingertip and arterial blood and their measurement variation. Clin Lab Haematol 23(3):155-159 